\title{
A hypomorphic inherited pathogenic variant in $D D X 3 X$ causes male intellectual disability with additional neurodevelopmental and neurodegenerative features
}

\author{
Georgios Kellaris ${ }^{1,2}$, Kamal Khan ${ }^{1,3}$, Shahid M. Baig ${ }^{3}$, I-Chun Tsai ${ }^{1}$, Francisca Millan Zamora ${ }^{6}$, Paul Ruggieri ${ }^{4}$,
} Marvin R. Natowicz ${ }^{5}$ and Nicholas Katsanis ${ }^{1,4^{*}}$ (D)

\begin{abstract}
Background: Intellectual disability (ID) is a common condition with a population prevalence frequency of 1-3\% and an enrichment for males, driven in part by the contribution of mutant alleles on the X-chromosome. Among the more than 500 genes associated with ID, DDX3X represents an outlier in sex specificity. Nearly all reported pathogenic variants of DDX3X are de novo, affect mostly females, and appear to be loss of function variants, consistent with the hypothesis that haploinsufficiency at this locus on the X-chromosome is likely to be lethal in males.

Results: We evaluated two male siblings with syndromic features characterized by mild-to-moderate ID and progressive spasticity. Quad-based whole-exome sequencing revealed a maternally inherited missense variant encoding p.R79K in DDX3X in both siblings and no other apparent pathogenic variants. We assessed its possible relevance to their phenotype using an established functional assay for DDX3X activity in zebrafish embryos and found that this allele causes a partial loss of DDX3X function and thus represents a hypomorphic variant.

Conclusions: Our genetic and functional data suggest that partial loss of function of DDX3X can cause syndromic ID. The p.R79K allele affects a region of the protein outside the critical RNA helicase domain, offering a credible explanation for the observed retention of partial function, viability in hemizygous males, and lack of pathology in females. These findings expand the gender spectrum of pathology of this locus and suggest that analysis for DDX3X variants should be considered relevant for both males and females.
\end{abstract}

\section{Background}

Intellectual disability (ID) is a common (estimated prevalence of $1-3 \%[1-3])$, clinically variable phenotype, defined by impairment in both intellectual function and adaptive behavior, with onset during the developmental period $[4,5]$. ID is clinically and etiologically heterogeneous, with varying disability of the two aspects of the condition [6]. In addition, ID can occur as an isolated trait

\footnotetext{
*Correspondence: natowim@ccf.org; nicholas.katsanis@duke.edu

${ }^{1}$ Center for Human Disease Modeling, Duke University, 300 North Duke

Street, Durham, NC 27701, USA

${ }^{4}$ Imaging Institute, Cleveland Clinic, 9500 Euclid Avenue, Cleveland, OH 44195, USA

Full list of author information is available at the end of the article
}

but is accompanied frequently by one or more comorbidities; these can include seizures or other neurological involvement [7], microcephaly or macrocephaly [8], dysmorphic features, and structural and/or functional abnormalities in organ systems other than the brain [9].

ID is more prevalent in males than females with a ratio of 1.3-1.4:1 [10], a gender bias partially reflective of the observation that numerous genes on the X-chromosome contribute to ID under an $\mathrm{X}$-linked recessive paradigm [10]. Indeed, substantial gene discovery efforts have focused on male-enriched or male-specific cohorts as a means to accelerate the discovery of ID-associated genes and mutational mechanisms [10]. Genetic and genomic 
studies of individuals, families, and populations with ID have led to the identification of more than 500 mutated genes, with some estimates predicting that number to exceed 1000, possibly reflective of the large number of transcripts expressed in and necessary for the development and maintenance of the central nervous system $[10,11]$. In several instances, female carriers are protected by virtue of functional mosaicism due to random $\mathrm{X}$-inactivation or by X-inactivation that favors the wildtype allele [12]. Further phenotypic analyses have also led to the appreciation of subtle phenotypic defects under a dosage model, in which males manifest the extreme range of pathology and females are partially protected [13].

The DDX3X locus on Xp11.4 represents an exception to the male specificity paradigm. To date, $D D X 3 X$ pathogenic variants have been reported in 35 females with ID $[3,14]$, all of which were de novo. As of September 2017, 52 cases have been deposited in Decipher: $49 \mathrm{fe}$ males, all but one of whom have de novo alleles (the 50th was inherited from a mosaic father) and three males, two harbor de novo mutations and one whose mutation is of unknown origin [15]. Consistent with the intolerance of the locus to variation [3], there are no null alleles found in control populations of either gender and a concomitant depletion of missense variants as well [16].

Herein, we report our investigation of two adult male siblings with ID, born to parents of normal intelligence. Whole-exome sequencing (WES) identified a rare, nonsynonymous, maternally transmitted variant in $D D X 3 X$ as a likely driver of their phenotype. DDX3X is necessary for canonical Wnt signaling [17], an observation utilized previously to develop an in vivo ventralization assay in zebrafish embryos as a means to assess the pathogenic potential of alleles [3]. Using this assay, we found that the $D D X 3 X$ variant results in reduced DDX3X function, reinforcing the genetic hypothesis. Together, our data support the notion that partial loss of function in $D D X 3 X$ can lead to ID in males and reinforce a gradient of severity model that is dependent on the strength of the mutation as it pertains to residual protein function.

\section{Materials and methods Subjects}

We studied a pedigree with two affected male siblings. Diagnostic testing of subjects was done as part of their clinical evaluations with informed consent from the mother and assent of the two cases. Research-based testing protocols were approved by the Cleveland Clinic Institutional Review Board.

\section{Whole-exome sequencing}

WES was carried out at GeneDx, Inc. subsequent to informed consent of the parents and assent of the affected individuals. Using genomic DNA from the ID cases and their parents, the exonic regions and flanking splice junctions of the genome were captured using the Clinical Research Exome kit (Agilent Technologies, Santa Clara, CA). Massively parallel (NextGen) sequencing was done on an Illumina system with 100 bp paired-end reads. The percent coverage at $10 \mathrm{X}$ or greater in the two brothers and their two parents ranged from 97.14 to 97.49\%; the younger brother had $97.18 \%$ coverage that was at least 10X and the older brother had $97.49 \%$ coverage at least at 10X. The mean coverage in the younger brother was $132 \mathrm{X}$ and in the older brother 197X. The mean coverage in the father and the mother was $134.29 \mathrm{X}$ and 222.05X, respectively.

\section{Data analysis}

Reads were aligned to human genome build GRCh37/ UCSC hg19 and were analyzed for sequence variants using a custom-developed analysis tool. Additional sequencing technology and variant interpretation protocols have been described [18]. The general assertion criteria for variant classification are available on the GeneDx ClinVar submission page (http://www.ncbi.nlm.nih.gov/clinvar/submitters/26957/). Variants of possible clinical significance relative to the phenotype of interest were confirmed by Sanger sequencing. Variants were named according to the following GenBank identifiers: DDX3X (NM_001356) and SPG7 (NM_003119).

\section{In vivo complementation studies in zebrafish}

We obtained a plasmid containing the wild-type (wt) human open reading frame (ORF) of $D D X 3 X$ and WNT3A from Ultimate ORF Collection (LifeTechnologies; clone ID DDX3X: IOH13891; WNT3A: IOH80731). Both plasmids were sequence-confirmed and cloned into the pCS2+ vector using Gateway LR clonase II-mediated recombination (LifeTechnologies) as described [19]. For $W N T 3 A$, a stop codon was introduced by site-directed mutagenesis using primer (5'-ctgcaaggccgccaggcacTAG GGTGGGCGCGCCGA-3' and its reverse complement). To generate the mutant constructs for the ID-associated variant c.236 G>A (p.R79K) along with the positive control female variant c.641T $>C \quad$ (p.I214T) and a negative control male variant c.898G $>\mathrm{T}$ (p.V300F), we conducted site-directed mutagenesis using primers (c.641T>C: 5' -CTATTCCTATTACCAAAGAGAAAAG3', c.898G>T: 5'CTAGAGTTCGTCCTTGCGTGTTT TATGGTGGTGCCGATATT-3', and c.236 G>A G: 5' TTGGATCTCGTAGTGATTCAAAAGGGAAGTCTAG CTTC-3' and their reverse complements). We then generated capped mRNA from linearized wt-DDX3X and WNT3A pCS2+ constructs as well as for $D D X 3 X$ variants with the mMessage mMachine SP6 kit (ThermoFisher). Injections were conducted with wt (ZDR) 
embryos, and resulting embryos were phenotyped at 48 hpf for eye phenotypes according to established criteria (class I: hypoplasia of the eye and class II: absence of one or both eyes; [3]. The mRNAs (wt-WNT3A, wt$D D X 3 X$, and $D D X 3 X$ variants) were injected in the yolk of the embryos at 1-4 cell stage as described [20]. We acquired lateral and dorsal images with an AZ100 florescent microscope (Nikon), digital sight black and white camera (Nikon), and NIS Elements software (Nikon) at $\times 4$ magnification. We determined statistical differences between pairs of batches with an unpaired $t$ test.

\section{Results}

\section{Clinical evaluations}

We evaluated two affected male siblings from a nonconsanguineous family (Fig. 1; Table 1; for full clinical descriptions, see Additional file 1). The eldest affected, currently 29 years old, was diagnosed with ID, macrocephaly, dysarthria, progressive spastic paraparesis, and decreased lower extremity strength. He presented for clinical evaluation at 2 years of age; he had reduced speech output for age and was easily agitated and hyperactive. He walked at 15 months, said his first words at 1-2 years, talked in sentences at 3-4 years, and was toilet-trained at 3 years. Developmental assessment at 6.5 years showed nonverbal cognitive function and composite mental processing at 5 and 3 percentiles, respectively, and adaptive behavior functions ranging from 0.3 to $3 \%$. At age 9.5 years, he showed verbal, performance, and full-scale IQ scores of 71, 80, and 73, respectively. Assessment of communication function showed receptive and expressive language function at about 0.5 and 0.04 percentiles, while adaptive behavior assessment showed communication, daily living, and socialization functions at 1,2, and 10 percentiles, respectively.

At 14 years of age, he developed a stiff gait. Exam at 15 years showed an acquired macrocephaly, an asymmetric spastic gait, lower extremity hyperreflexia, and tight heel cords. He began treatment with oral baclofen for spasticity at 17 years of age. He had an adjusted curriculum during high school, graduated at 19 years, and has worked part-time afterwards in a supported workplace. His gait slowly worsened and he was last able to ambulate independently at 21 years. On neurological exam at 23 years, there was a slow, spastic gait, decreased lower extremity strength, especially with hip flexion, markedly increased the tone of the lower extremities, lower extremity hyperreflexia, and slowed fine finger and rapid alternating movements. At 29 years of age, he has progressive lower extremity and hand weakness and decreased fine motor function.

Brain MRI scan at 16 years of age (Fig. 1i) showed mildly prominent lateral ventricles and cortical sulci, atrophy of the entire corpus callosum that was accentuated in the anterior body and genu, and mild, symmetric, confluent T2-hyperintensity in the supratentorial periventricular white matter. The follow-up study 2 years later demonstrated further interval enlargement of the lateral ventricles and periventricular white matter T2hyperintensity, suggesting progression of central white matter volume loss. Myelination was otherwise within normal limits (Fig. 1ii). Cerebrospinal fluid analyses at 19 years showed a normal cell count, CSF glucose, and protein levels and normal CSF IgG synthesis and index. Metabolic testing included increased CSF alanine and intermittent mildly increased urinary lactate (Additional file 2: Table S1).

The younger affected brother, 25 years old, has progressive spastic paraparesis and tremor and learning disability/mixed expressive-receptive language disorder (Fig. 1; Table 1). The first concern about his neurodevelopmental status was when he was about 2 years old and had mildly delayed speech development; he spoke in sentences at 3 years and began speech therapy at that time. He developed an intentional hand tremor at about 6 years that worsened over time. Developmental assessment at 5.6 years showed normal development in all areas except for mild receptive and expressive language delay. Re-evaluation at 6 years showed receptive and expressive language skills at 39 and 16 percentiles, with weaknesses in auditory memory, interpreting directions, and categorizing words. Assessment at 7 years showed verbal, performance, and full-scale IQ scores of 84, 81, and 81 , respectively. Academic achievement testing showed basic reading, reading comprehension, mathematics calculation, mathematics reasoning, and written expression at 1, 5, 16, 9, and 23 percentiles, respectively; receptive and expressive language tested at 37 and 16 percentiles. He did not have significant behavioral issues.

Examination at 15 years old, for worsening hand tremors, showed a non-dysmorphic male with lower extremity hyperreflexia and tremor; the gait and motor tone were normal. At 17 years, he was noted to have a mild spastic gait and bilateral Babinski signs. At 18 years, he was noted to have a worsening asymmetric spastic paretic gait and bilateral hip flexor weakness. Schooling involved an adjusted curriculum with training in service occupations, and he graduated from high school at 18 years of age, subsequently working part-time and requiring supports in complex daily living tasks.

His diagnostic evaluation included an abnormal brain MRI scans at 15,16 , and 17 years of age showing mild, diffuse prominence of the cortical sulci suggesting mild cortical volume loss and mild enlargement of the lateral ventricles, generalized volume loss in the corpus callosum that was more prominent in the genu and anterior body, and mild symmetric confluent periventricular T2- 

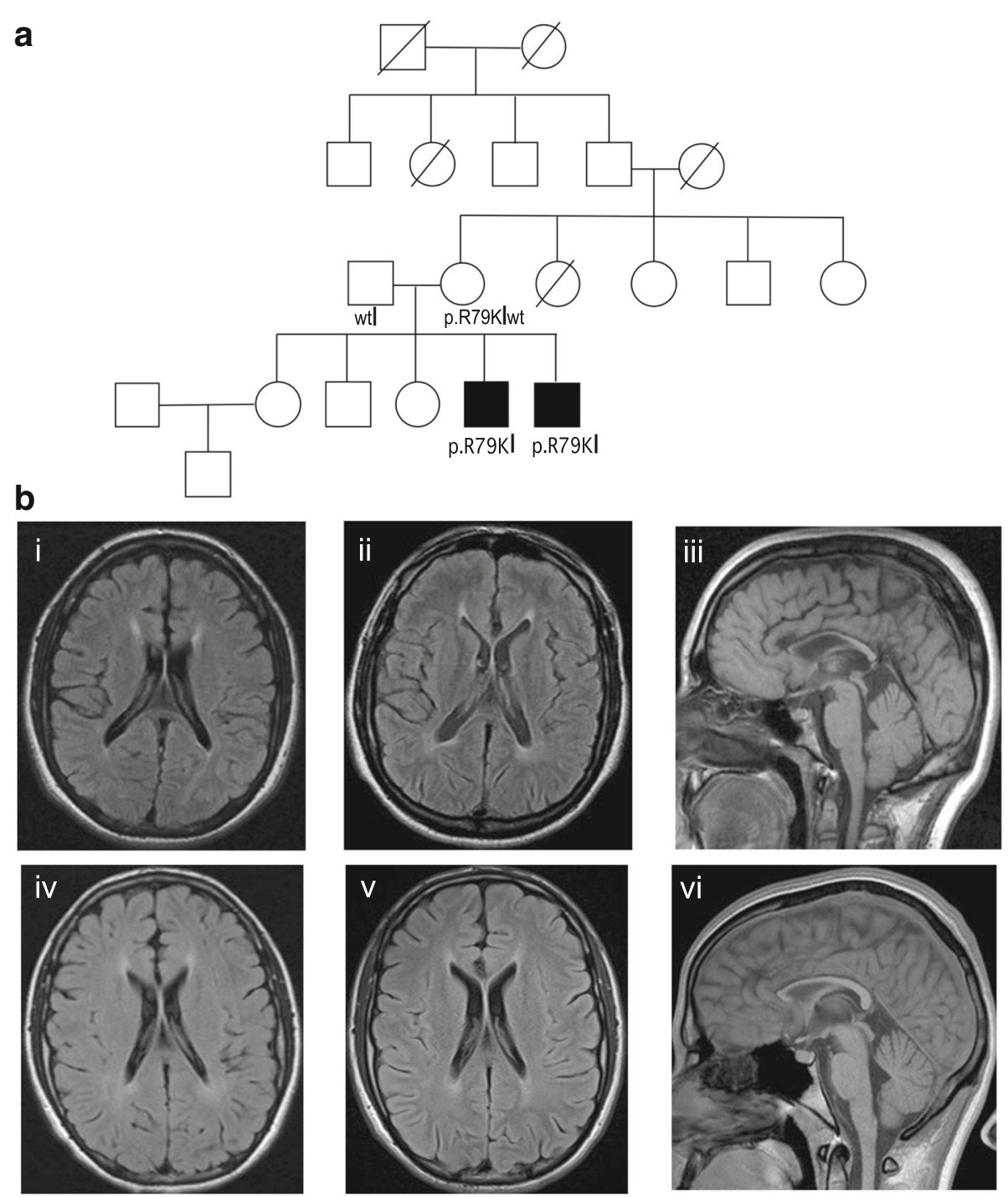

Fig. 1 Brain MRI of both affected syndromic ID cases. a Family pedigree showing the two affected brothers and the family pedigree. b (i) Axial T2 FLAIR of sibling 1 at age 16 demonstrates mild enlargement of the lateral ventricles and mild confluent hyperintensity in the adjacent white matter. (ii) Axial T2 FLAIR of sibling 12 years later demonstrates interval enlargement of the lateral ventricles and mild progression of the confluent hyperintensity in the adjacent white matter suggesting progressive damage to the central white matter and volume loss. (iii) Sagittal T1 demonstrates generalized volume loss in the corpus callosum that is more prominent in the genu and anterior body, further supporting central white matter volume loss that is more severe anteriorly. (iv) Axial T2 FLAIR of sibling 2 at age 15 demonstrates mild enlargement of the lateral ventricles and mild, symmetric hyperintensity in the adjacent white matter, suggesting central white matter volume loss and gliosis. (v) Axial $T 2$ FLAIR of sibling 22 years later demonstrates mild interval increase in size of the lateral ventricles suggesting mild progression of central white matter volume loss, but no significant change in the periventricular hyperintensity. (vi) Sagittal T1 image is also comparable in appearance to his sibling, with prominent volume loss in the corpus callosum that is more striking anteriorly

hyperintensity within the supratentorial periventricular white matter suggesting central white matter volume loss that was more prominent in the frontal lobes (Fig. 1iv-vi). There was also slight further enlargement of the lateral ventricles without significant change in the T2-hyperintensity or the corpus callosum over the 2-year period. The MRI of the entire spinal cord was normal in caliber and signal intensity. CSF analyses showed normal cellular CSF and normal levels of CSF glucose and protein, normal CSF IgG synthesis and IgG index, normal levels of CSF lactate and pyruvate, and increased CSF alanine. Plasma amino acid analyses consistently showed mildly moderately increased levels of alanine and, sometimes, increased levels of proline and glycine (Additional file 2: Table S1).

\section{Genomic analysis}

A host of known genetic and metabolic etiologies of ID and of spasticity were ruled-out in the two cases 
Table 1 Summary of clinical findings in the two subjects. Human phenotype ontology (HPO) terms and codes are shown

\begin{tabular}{|c|c|c|c|}
\hline Phenotype & HPO code & Case 1 & Case 2 \\
\hline Intellectual disability or related neurodevelopmental disability & HP:0001256 & + & + \\
\hline Macrocephaly & HP:0000256 & + & + \\
\hline Dysarthria & HP:0001260 & + & + \\
\hline Tight heel cords & & + & + \\
\hline Progressive spastic paraparesis & HP:0007199 & + & + \\
\hline Tremor & HP:0002322 & - & + \\
\hline Hand weakness & HP:0030237 & + & - \\
\hline Proximal leg weakness & HP:0007340 & + & + \\
\hline Brain MRI with abnormal periventricular T2 intensity & HP:0002518 & + & + \\
\hline Ventriculomegaly & HP:0002119 & + & + \\
\hline Atrophy of corpus callosum, esp. the genu and anterior body of corpus callosum & HP:0006989 & + & + \\
\hline Increased cerebrospinal fluid alanine level & $\mathrm{n} / \mathrm{a}$ & + & + \\
\hline
\end{tabular}

n/a not applicable

(Additional file 2: Table S1). Consequently, given that both parents are of normal intelligence, we undertook quad-based WES analysis hypothesizing either a recessive or an X-linked paradigm of disease inheritance. Under a rare variant hypothesis, the WES data were evaluated for alleles shared among the two affected siblings that are rare (minor allele frequency $<1 \%$ ) and affect coding residues or canonical splice sites. We identified several variants of potential interest (Additional file 3: Table S2), but only a single, maternally inherited variant fulfilled our stringent criteria: a c. $236 \mathrm{G}>\mathrm{A}$ change encoding p.R79K in $D D X 3 X$ was identified (Fig. 2a, b). The $D D X 3 X$ locus has been implicated previously in ID and, sometimes, other features in females; however, nearly all published variants described to date are de novo, which has led to the hypothesis that haploinsufficiency of $D D X 3 X$ in males might be incompatible with life [3]. Nonetheless, multiple sequence alignment across vertebrates showed the p.R79 position to be conserved (Fig. 2b). Prediction algorithms were discordant: PolyPhen2 [21] predicted the allele to be benign, whereas MutationTaster [22] predicted it to be deleterious. Overall, 11 variant prediction algorithms predict this

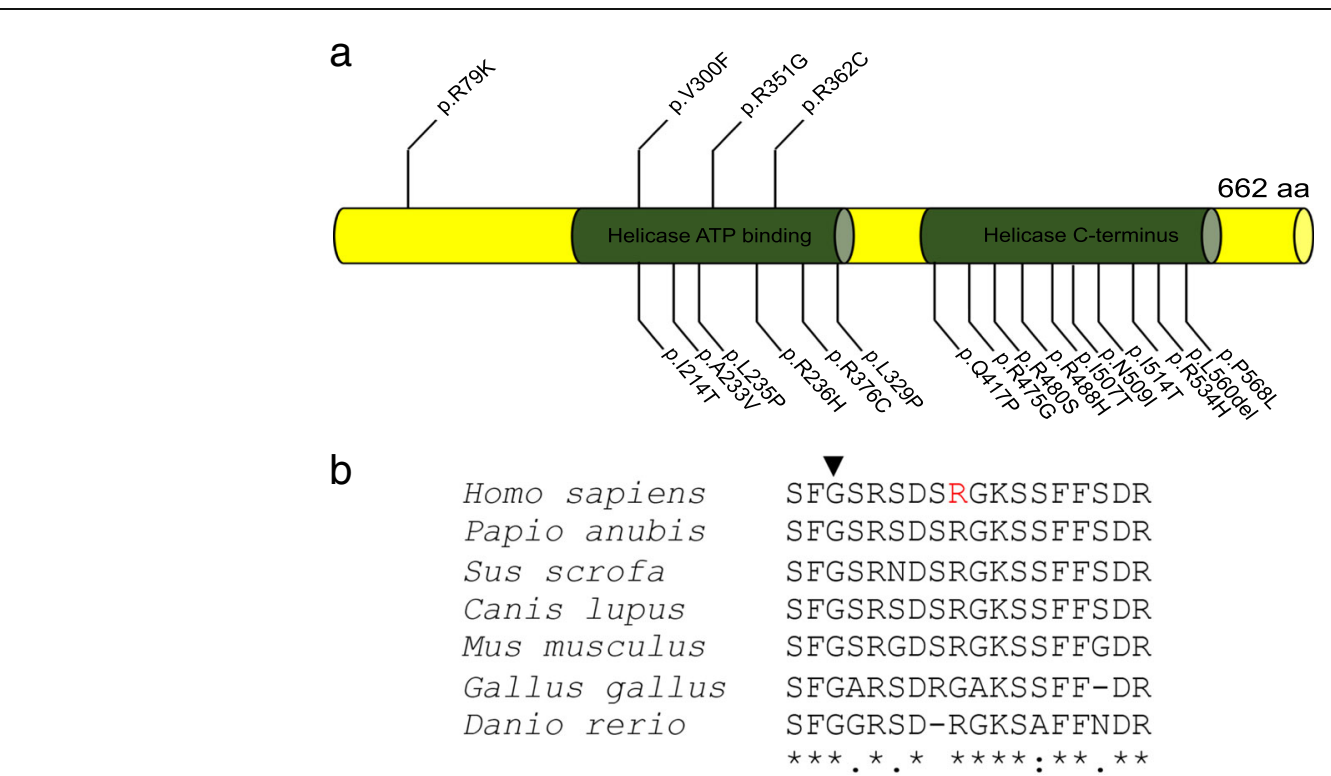

Fig. 2 Discovery of a maternally transmitted DDX3X variant in male ID. a Protein sequence alignment of DDX3X across vertebrate species; the mutated residue is shown by arrow. $\mathbf{b}$ Location of all functionally tested amino acid substitutions in DDX3X. Reported male alleles (top); alleles found in females (bottom). The helicase ATP-binding domain and a helicase C-terminal domain are also shown (green) 
variant to be damaging to the structure/function of DDX3X out of a total of 21 in silico assessments [23], http://159.226.67.237/sun/varcards/welcome/index). The variant was found only once in heterozygosity, in a female, in $>170,000$ control exomes [16]. We note that, in contrast to the majority of described $D D X 3 X$ variants that lie in the helicase domain, the p.R79K variant maps proximal to that region (Fig. 2b). Together, these data raised the hypothesis that, in contrast to previously reported alleles in $D D X 3 X$ that abrogate protein function, p.R79K might be a hypomorph, which could explain its transmission and the phenotypic outcome in this kindred.

\section{Functional testing}

We and others have shown that expression of human $D D X 3 X$ mRNA exacerbates the ventralization phenotype induced by the expression of femtogram ( $\mathrm{fg}$ ) quantities of the canonical Wnt ligand WNT3A in zebrafish embryos [3]. This phenotype can be binned into two phenotypic classes (class I and class II; Fig. 3a-c) based on previously established and validated morphometric criteria [24]. Using this model system, we tested the functionality of missense $D D X 3 X$ alleles discovered in ID patients and found that DNA sequence variants predicted to cause loss of function of $D D X 3 X$ and which were associated with ID failed to exacerbate WNT3A-driven pathology, whereas benign alleles were indistinguishable in their activity and promoted significant Wnt-dependent pathology [3]. Here, we utilized this assay to test the activity of the p.R79K allele. First, we optimized the in vivo complementation assays to the $Z D R$ wt genetic background by injecting progressively increasing doses of wt-WNT3A; we found that $550 \mathrm{fg}$ of mRNA was sufficient to induce a modest phenotype $(\sim 8-15 \%$ affected embryos, $n=50-100$ embryos per clutch, replicated; Fig. 3, Additional file 4: Figure S1, Additional file 5: Figure S2A). On this background sensitization with $W N T 3 A$, we titrated the amount of human $D D X 3 X$ mRNA required to generate pathology. We found a dose-dependent effect that was maximal at $30 \mathrm{pg}$ mRNA (Additional file 4: Figure S1). Therefore, we selected the $500 \mathrm{fg} / 30 \mathrm{pg}$ mRNA doses to test the effect of the discovered allele. Next, we co-injected WNT3A-sensitized $Z D R$ embryos with wt $D D X 3 X, D D X 3 X$ encoding the candidate pathogenic allele p.R79K, the known benign allele p.V300F, and the known pathogenic allele p.I214T. Upon blind scoring to injection cocktail, both the positive and negative controls scored as expected: mRNA encoding p.300F was indistinguishable from wt, whereas p.214T failed to induce any significant pathology exceeding that of WNT3A alone, consistent with a null (or near null) allele. The candidate mutation of interest, p.R79K, scored intermediate, with the extent of pathology of the resulting embryos significantly different from both wt and null $D D X 3 X(p<$ 0.0008 and $p<0.01$ respectively; Fig. $3 \mathrm{~d}$ ). In contrast, expression of either wt or mutant $D D X 3 X$ mRNA alone did not induce any appreciable pathologies, likely excluding any dominant negative mechanisms (Additional file 5: Figure S2B). Together with the human genetics and evolutionary conservation, these data suggest that the discovered allele is a likely hypomorph.
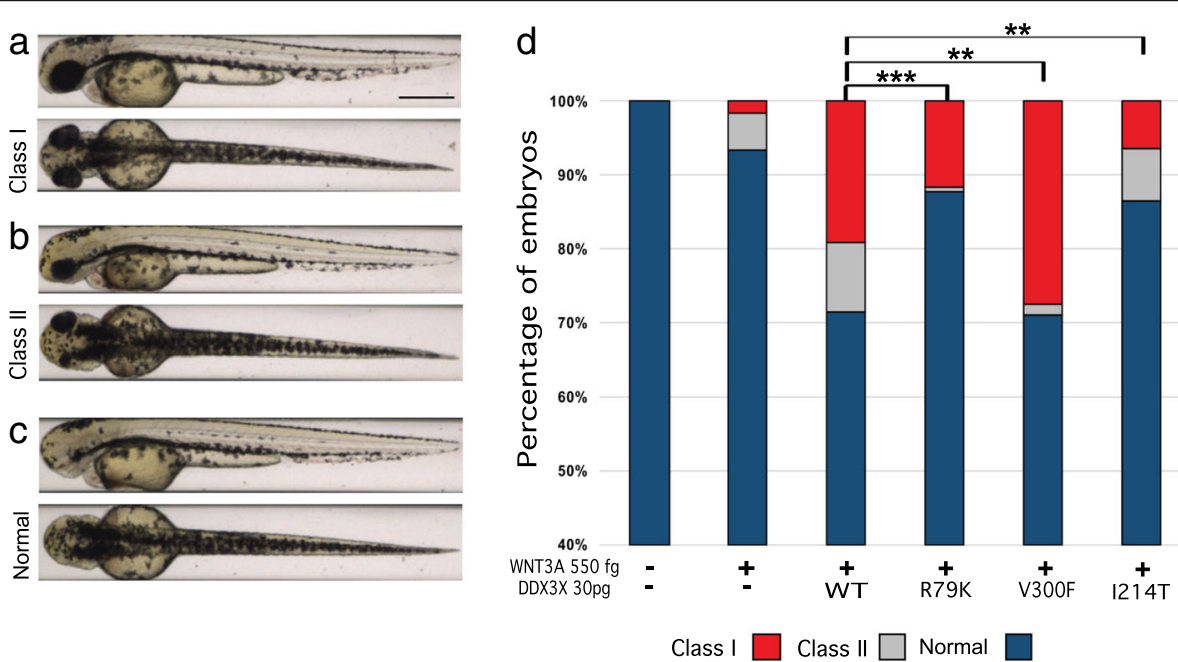

Fig. 3 Functional testing of DDX3X variants. a-c Representative lateral images of zebrafish embryos at 2 dpf that are either uninjected (a) or injected with human WNT3A without (b) or with (c) human DDX3X show a range of ventralized phenotypes. These were scored according to established criteria as normal, class I, or class II ventralization. No injection condition resulted in severe ventralization (class III or IV) [3]. d DDX3X variants were tested for their effect on increasing WNT3A-mediated ventralization using $550 \mathrm{fg}$ of WNT3A mRNA and 30 pg of DDX3X mRNA per embryo. $P$ values: $<0.0001$ (four asterisks); 0.0001 to 0.001 (three asterisks); 0.001 to 0.01 (two asterisks); 0.01 to 0.05 (one asterisk); $\geq 0.05$ not significant (ns) 


\section{Discussion}

Here, we describe the clinical phenotypes of two adult males who have a rare, maternally inherited missense allele of $D D X 3 X$, a major ID locus that to date has been associated with pathology almost exclusively in females. Both genetics and functional testing support a partial loss of function disease transmission. This notion is consistent with the observation that the mother who transmitted the p.R79K is asymptomatic and that the two affected males have significant neurodevelopmental pathology but have survived. Although the formal possibility remains that the mother might exhibit sub-clinical neuroanatomical findings, careful re-examination revealed no overt neurological concerns. In addition to this notion, it would be important to identify the genotype for the maternal uncle and maternal grandfather, but DNA samples from the individuals were not available. This hypothesis is also supported by recent work in the mouse revealing essential roles of $D d x 3 x$ in placentation and embryogenesis and the differential phenotypic impacts of inheriting a paternal vs. maternal null allele [25]. Non-lethality of the p.R79K variant in our cases may be due to the maternal, as opposed to paternal, inheritance of the variant. Nonlethality of the p.R79K variant might also be because the location of the amino acid substitution is outside of the critical RNA helicase domain. The DDX3X protein has been investigated extensively [26], but the region of the protein surrounding amino acid residue 79 has only been studied sparingly, although sitedirected mutagenesis of several nearby residues resulted in functional impacts [27, 28]. Finally, nonlethality of the p.R79K variant might be explained on the basis of the expected moderate biophysical impact on the structure of DDX3X consequent to the conservative nature of an arginine to lysine substitution.

There is substantial clinical and neuroradiologic overlap between the phenotype in our two cases and those of the females and few males described to date. The most common traits described thus far include ID, microcephaly, hypotonia, movement disorder and/or spasticity, ventricular enlargement, and hypoplasia of the corpus callosum; the subjects reported here have ID, spasticity, ventricular enlargement, and an attenuated corpus callosum. Our two cases also present with features that are distinct from those of other cases with variants in $D D X 3 X$ reported to date; these include progressive spasticity with loss of independent ambulation and, consistent with their clinical courses, evidence of a progressive brain central white matter process including atrophy of the corpus callosum, not hypoplasia. Long-term follow-up of the previously reported cases, some of whom were young children, will be important to determine if any of the neurological findings or developmental disabilities are progressive and if there are ongoing brain white matter changes.

It is also possible that a second clinical process that is distinct from DDX3X may be present in the two subjects reported here. In this regard, both subjects have macrocephaly, as does their father and one sister although the father and the subjects' siblings have clinical phenotypes that are otherwise disparate from the clinical findings in the cases with ID. The finding of increased levels of CSF alanine in both cases and of increased plasma alanine in one case and increased urinary lactate in the other is notable and suggests a disturbance of mitochondrial bioenergetics. No evidence of a primary mitochondrial cytopathy was found, despite considerable investigation, and the relationship of these results to the variant of $D D X 3 X$ is uncertain. In addition, the existence of a clinical process that is/was present in only one of the subjects is likely in view of the variability in neurodevelopmental phenotype between the subjects, although the basis of this process is unknown. Given the reported spasticity in this pedigree, we did evaluate known spastic paraplegia genes for candidate pathogenic variants. We detected a maternally inherited heterozygous variant in SPG7 in one of the subjects that has been reported to be pathogenic in patients with recessive spastic paraplegia [29]. Although this allele is by itself unable to explain the observed spasticity in our patient, it is possible that it contributes to the phenotype, possibly in epistasis with $D D X 3 X$; the same might be true for some of the other ultra-rare alleles discovered in this family (Additional file 3: Table S2). For example, it is intriguing that the older brother with the more extensive pathology is also hemizygous for candidate pathogenic variant in ARGHGAP4, a gene whose product is required for neuronal migration and which has been reported to be mutated in some patients with ID [30-32]. However, both formal functional testing and delineation of additional patients will be necessary to test this hypothesis.

\section{Conclusions}

Overall, our findings suggest that mutational analysis of $D D X 3 X$ in either genetic testing panels or by wholeexome/genome sequencing should not be limited, by testing or interpretation of data, to females. The severity of most discovered $D D X 3 X$ alleles remains consistent with a model of total haploinsufficiency incompatible with life in males. Further studies in expanded cohorts will illuminate this proposed phenotype-genotype correlation further.

\section{Additional files}

Additional file 1: Detailed Clinical report. (DOCX $23 \mathrm{~kb}$ ) 
Additional file 2: Table S1. Clinical Laboratory Diagnostic Evaluations. (DOCX $16 \mathrm{~kb})$

Additional file 3: Table S2. Sequence variants identified according to mode of inheritance. (DOCX $16 \mathrm{~kb}$ )

Additional file 4: Figure S1. Co-injection of DDX3X and WNT3A mRNA produces dose-dependent changes in ventralization. Zebrafish embryos were injected with $550 \mathrm{fg}$ WNT3A mRNA without or with wt DDX3X mRNA at varying doses. Embryos were scored at $48 \mathrm{~h}$ post fertilization for degree of ventralization according to described objective criteria. $P$ value: $<0.0001,\left({ }^{* * *}\right) ; 0.0001$ to $0.001,\left({ }^{* *}\right) ; 0.001$ to $0.01,\left(^{* *}\right) ; 0.01$ to $0.05,(*) ; \geq 0.05$, not significant (ns). (PDF $1597 \mathrm{~kb})$

Additional file 5: Figure S2. Dose-response effect of WNT3A and overexpression of DDX3X variants does not produce alterations in Wnt signaling. (A) Injection of WNT3A produces dose-dependent changes in ventralization. Embryos were scored at $48 \mathrm{~h}$ post fertilization for degree of ventralization. (B) Zebrafish embryos were injected with $100 \mathrm{pg}$ of DDX3X mRNA containing either the wild-type sequence or the non-synonymous variants found in the affected individuals. At $36 \mathrm{~h}$ post-fertilization (hpf) the embryos were phenotyped; no abnormalities could be appreciated. (PDF $1438 \mathrm{~kb}$ )

\section{Acknowledgements}

We thank the family for the participation and encouragement of this work. We thank Erica Davis for the critical evaluation of the manuscript and data.

\section{Funding}

NK is a Brumley Distinguished Professor of Pediatrics. The study was supported by Core Funding from the Center for Human Disease Modeling at Duke University.

\section{Availability of data and materials}

All material on this paper is available for distribution subject to appropriate regulatory clearance.

\section{Authors' contributions}

GK and KK performed experiments, reviewed and compiled data, and co-wrote the paper. SMB and ICT and NK reviewed and interpreted the data and provided guidance to the work. MRN assessed the family, reviewed and interpreted the WES data, and co-wrote the manuscript. FMZ reviewed and interpreted WES. PR reviewed the brain MRIs. All authors reviewed and commented on the manuscript. All authors read and approved the final manuscript.

\section{Ethics approval and consent to participate}

The study was approved by Cleveland Clinic Institutional Review Board protocol \#06-333 review board, and the family was enrolled in the study under informed consent, including consent to publish the study's findings. All experiments involving the use of zebrafish (Danio rerio) were approved by the Duke University Institutional Animal Care and Use Committee (IACUC).

\section{Consent for publication}

Written informed consent for publication of clinical details and clinical images was obtained from the parent of the probands.

\section{Competing interests}

NK is a paid consultant and holds founding stock in Rescindo Therapeutics.

\section{Publisher's Note}

Springer Nature remains neutral with regard to jurisdictional claims in published maps and institutional affiliations.

\section{Author details}

${ }^{1}$ Center for Human Disease Modeling, Duke University, 300 North Duke Street, Durham, NC 27701, USA. 'Department of Medical Genetics, University of Athens Medical School, Aghia Sophia Children's Hospital, 11527 Athens,
Greece. ${ }^{3}$ Human Molecular Genetics Laboratory, Health Biotechnology Division, National Institute for Biotechnology and Genetic Engineering (NIBGE), Faisalabad 38000, Pakistan. ${ }^{4}$ Imaging Institute, Cleveland Clinic, 9500 Euclid Avenue, Cleveland, OH 44195, USA. ${ }^{5}$ Pathology and Laboratory Medicine and Genomic Medicine Institutes, Cleveland Clinic, Cleveland, $\mathrm{OH}$ 44195, USA. 'GeneDx, 207 Perry Parkway, Gaithersburg, MD 20877, USA.

Received: 30 November 2017 Accepted: 15 February 2018

Published online: 01 March 2018

\section{References}

1. Wolfe K, Stueber K, McQuillin A, Jichi F, Patch C, Flinter F, Strydom A, Bass N Genetic testing in intellectual disability psychiatry: opinions and practices of UK child and intellectual disability psychiatrists. J Appl Res Intellect Disabil. 2017;31(2):273-84

2. van Bokhoven $\mathrm{H}$. Genetic and epigenetic networks in intellectual disabilities. Annu Rev Genet. 2011;45:81-104

3. Snijders Blok L, Madsen E, Juusola J, Gilissen C, Baralle D, Reijnders MR, Venselaar H, Helsmoortel C, Cho MT, Hoischen A, et al. Mutations in DDX3X are a common cause of unexplained intellectual disability with genderspecific effects on Wnt signaling. Am J Hum Genet. 2015;97(2):343-52.

4. Harris JC. Intellectual disability: Understanding its development, causes, classification, evaluation, and treatment. New York: Oxford University Press; 2006.

5. Tasse MJ, Luckasson R, Nygren M. AAIDD proposed recommendations for ICD-11 and the condition previously known as mental retardation. Intellect Dev Disabil. 2013;51(2):127-31.

6. American Psychiatric Association. Diagnostic and statistical manual of mental disorders: DSM-5: Fifth edition. Arlington: American Psychiatric Publishing; 2013.

7. McGrother CW, Bhaumik S, Thorp CF, Hauck A, Branford D, Watson JM. Epilepsy in adults with intellectual disabilities: prevalence, associations and service implications. Seizure. 2006;15(6):376-86.

8. de Ligt J, Willemsen MH, van Bon BW, Kleefstra T, Yntema HG, Kroes T, Vulto-van Silfhout AT, Koolen DA, de Vries P, Gilissen C, et al. Diagnostic exome sequencing in persons with severe intellectual disability. N Engl J Med. 2012;367(20):1921-9.

9. Matson JL, Cervantes PE. Comorbidity among persons with intellectual disabilities. Res Autism Spectr Disord. 2013;7(11):1318-22.

10. Vissers LE, Gilissen C, Veltman JA. Genetic studies in intellectual disability and related disorders. Nat Rev Genet. 2016;17(1):9-18.

11. Kochinke K, Zweier C, Nijhof B, Fenckova M, Cizek P. Honti F, Keerthikumar S, Oortveld MA, Kleefstra T, Kramer JM, et al. Systematic phenomics analysis deconvolutes genes mutated in intellectual disability into biologically coherent modules. Am J Hum Genet. 2016;98(1):149-64.

12. Cotton AM, Price EM, Jones MJ, Balaton BP, Kobor MS, Brown CJ. Landscape of DNA methylation on the $X$ chromosome reflects $\mathrm{CpG}$ density, functional chromatin state and X-chromosome inactivation. Hum Mol Genet. 2015:24(6):1528-39.

13. Fieremans $\mathrm{N}$, Van Esch $\mathrm{H}$, Holvoet M, Van Goethem G, Devriendt K, Rosello M, Mayo S, Martinez F, Jhangiani S, Muzny DM, et al. Identification of intellectual disability genes in female patients with a skewed X-inactivation pattern. Hum Mutat. 2016:37(8):804-11.

14. Dikow N, Granzow M, Graul-Neumann LM, Karch S, Hinderhofer K Paramasivam N, Behl L, Kaufmann L, Fischer C, Evers C, et al. DDX3X mutations in two girls with a phenotype overlapping Toriello-Carey syndrome. Am J Med Genet A. 2017;173(5):1369-73.

15. Firth HV, Richards SM, Bevan AP, Clayton S, Corpas M, Rajan D, Vooren SV, Moreau Y, Pettett RM, Carter NP. DECIPHER: database of chromosoma imbalance and phenotype in humans using ensembl resources. Am J Hum Genet. 2009;84(4):524-33.

16. Lek M, Karczewski K, Minikel EV, Samocha KE, Banks E, Fennell T, O'DonnellLuria AH, Ware JS, Hill AJ, Cummings BB, et al. Analysis of protein-coding genetic variation in 60,706 humans. Nature. 2016;536:285

17. Cruciat $C M$, Dolde $C$, de Groot RE, Ohkawara B, Reinhard C, Korswagen HC, Niehrs C. RNA helicase DDX3 is a regulatory subunit of casein kinase 1 in Wnt-beta-catenin signaling. Science. 2013;339(6126):1436-41.

18. Tanaka AJ, Cho MT, Millan F, Juusola J, Retterer K, Joshi C, Niyazov D, Garnica A, Gratz E, Deardorff M, et al. Mutations in SPATA5 are associated with microcephaly, intellectual disability, seizures, and hearing loss. Am J Hum Genet. 2015;97(3):457-64. 
19. Niederriter AR, Davis EE, Golzio C, Oh EC, Tsai IC, Katsanis N. In vivo modeling of the morbid human genome using Danio rerio. J Vis Exp. 2013;78:e50338.

20. Davis $\mathrm{EE}$, Frangakis $\mathrm{S}$, Katsanis N. Interpreting human genetic variation with in vivo zebrafish assays. Biochim Biophys Acta. 2014;1842(10):1960-70.

21. Adzhubei IA, Schmidt S, Peshkin L, Ramensky VE, Gerasimova A, Bork P, Kondrashov AS, Sunyaev SR: A method and server for predicting damaging missense mutations. In: Nat Methods. 2010;7:248-9.

22. Schwarz JM, Cooper DN, Schuelke M, Seelow D. MutationTaster2: mutation prediction for the deep-sequencing age. Nat Methods. 2014;11:361.

23. Li M, Gong L, Zhao D, Zhou J, Xiang H. The spacer size of I-B CRISPR is modulated by the terminal sequence of the protospacer. Nucleic Acids Res. 2017:45(8):4642-54.

24. Liu YP, Tsai IC, Morleo M, Oh EC, Leitch CC, Massa F, Lee BH, Parker DS, Finley D, Zaghloul NA, et al. Ciliopathy proteins regulate paracrine signaling by modulating proteasomal degradation of mediators. J Clin Invest. 2014;124(5):2059-70.

25. Chen C-Y, Chan C-H, Chen C-M, Tsai Y-S, Tsai T-Y, Wu Lee Y-H, You L-R. Targeted inactivation of murine Ddx3x: essential roles of Ddx3x in placentation and embryogenesis. Hum Mol Genet. 2016;25(14):2905-22.

26. Sharma D, Jankowsky E. The Ded1/DDX3 subfamily of DEAD-box RNA helicases. Crit Rev Biochem Mol Biol. 2014:49(4):343-60.

27. Gu L, Fullam A, Brennan R, Schroder M. Human DEAD box helicase 3 couples IkappaB kinase epsilon to interferon regulatory factor 3 activation. Mol Cell Biol. 2013;33(10):2004-15.

28. Oda S, Schroder M, Khan AR. Structural basis for targeting of human RNA helicase DDX3 by poxvirus protein K7. Structure. 2009;17(11):1528-37.

29. Elleuch N, Depienne C, Benomar A, Hernandez AM, Ferrer X, Fontaine B, Grid D, Tallaksen CM, Zemmouri R, Stevanin G, et al. Mutation analysis of the paraplegin gene (SPG7) in patients with hereditary spastic paraplegia. Neurology. 2006;66(5):654-9.

30. Vogt DL, Gray CD, Young WS 3rd, Orellana SA, Malouf AT. ARHGAP4 is anovel RhoGAP that mediates inhibiotion of cell motility and axonal outgrowth. Mol Cell Neurosci. 2007;36(3):332-42.

31. Liu F, Guo H, Ou M, Hou X, Sun G, Gong W, Jing H, Tan Q, Xue W, Dai Y, Su W. ARHGAP4 mutated in a Chinese intellectually challenged family. Gene. 2016:578(2):205-9.

32. Huang L, Poke G, Gecz J, Gibson K. A novel contiguous gene deletion of AVPR2 and ARHGAP4 genes in male dizygotic twins with nephrogenic diabetes insipidus and intellectual disability. Am J Med Genet A. 2012:158a(10):2511-8.

\section{Submit your next manuscript to BioMed Central and we will help you at every step:}

- We accept pre-submission inquiries

- Our selector tool helps you to find the most relevant journal

- We provide round the clock customer support

- Convenient online submission

- Thorough peer review

- Inclusion in PubMed and all major indexing services

- Maximum visibility for your research

Submit your manuscript at www.biomedcentral.com/submit

) Biomed Central 